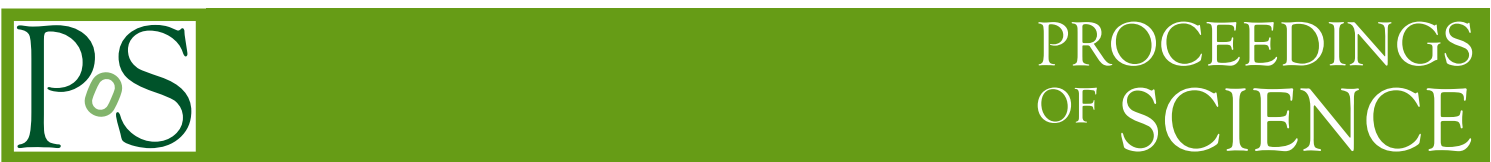

\title{
Recurrent Novae in the Local Group
}

\author{
Marina Orio* \\ INAF-Padova, vicolo Osservatorio 5, 35122 Padova, Italy, and Department of Astronomy, 475 \\ N. Charter Str., University of Wisconsin, Madison WI 53706, USA \\ E-mail: marina.oriodoapd.inaf.it
}

\begin{abstract}
Recurrent novae are those novae known to have had at least two outbursts within a century. I review here the main characteristics of these objects in the Local Group, selecting criteria for a basic classification, and discussing both the state of theoretical modeling and initial statistics in external galaxies. Generally, recurrent novae in short period systems with unevolved companions have quite slow outbursts, whereas the outbursts in RN with evolved secondaries evolve very quickly, and there is evidence that they host very massive white dwarfs. I discuss in detail some of the X-ray gratings observations of recurrent novae in the Local Group, demonstrating what can be learned from them. The most important parameter that can be derived is the effective temperature of the white dwarf, which is directly connected with its mass.
\end{abstract}

The Golden Age of Cataclysmic Variables and Related Objects - III, Golden2015

7-12 September 2015

Palermo, Italy

${ }^{*}$ Speaker. 
Table 1: List of Galactic Recurrent Novae, their maximum and minimum visual magnitudes, time for a decay by 3 magnitudes in optical, orbital period, and outburst years.

\begin{tabular}{lccccc} 
Name & $\mathrm{V}_{\text {peak }}(\mathrm{mag})$ & $\mathrm{V}_{\text {peak }}(\mathrm{min})$ & $\mathrm{t}_{3}$ (days) & $\mathrm{P}_{\text {orb }}$ (days) & Eruption years \\
\hline T Pyx & 6.4 & 15.5 & 62 & 0.076 & $1890,1902,1920,1944,1967,2011$ \\
IM Nor & 8.5 & 18.3 & 80 & 0.102 & 1920,2002 \\
CI Aql & 9.0 & 16.7 & 32 & 0.62 & $1917,1941,2000$ \\
V2487 Oph & 9.5 & 17.3 & 8 & - & 1900,1998 \\
U Sco & 7.5 & 17.6 & 2.6 & 1.23 & $1863,1906,1917,1936,1945,1969,1979,1987,1999,2010$ \\
V394 CrA & 7.2 & 18.4 & 5.2 & 1.52 & 1949,1987 \\
T CrB & 2.5 & 9.8 & 6 & 228 & 1866,1946 \\
RS Oph & 4.8 & 11 & 14 & 457 & $1898,1907,1933,1945,1958,1967,1985,2006$ \\
V745 Sco & 9.4 & 18.6 & 9 & 510 & 1962,1990 \\
V3890 Sgr & 8.1 & 15.5 & 14 & 519.7 & 0 \\
\hline & & & & &
\end{tabular}

\section{The Galactic Recurrent Novae}

There are only 10 known Galactic recurrent novae (hereafter, $\mathrm{RN}$ ), although repeated outburst may have occurred when a given nova was not observable. In fact most RN are quite fast, and return to minimum light within few months. Table 1 lists the known Galactic RN, with their peak and minimum magnitude, the time for a decay by 3 magnitudes $t_{3}$, the orbital period and the years of observed outbursts. Most details can be found already in Schaefer (2010). The common characteristics are:

- The measured recurrence time of less than 100 years;

- A visual outburst amplitude of not more than $11 \mathrm{mag}$ and generally around 7-8 mag, which is on average quite smaller than that of other ("classical") novae,

- A classification of He/N optical spectral class. Williams, who proposed the classification of Fe II vs. He/N novae, later proposed that these are only "spectral phases" and that the Fe II phase is very short in RN (Williams 2012). This may explain why IM Nor, the slowest RN, is an outlier in this respect, having shown a Fe II type spectrum.

In general RN seem to be outliers in the maximum magnitude versus rate of decline (MMRD) relationship, so it is difficult to derive their distances. Another inferred characteristic is that the rate of mass transfer $\dot{m}$ onto the white dwarf (hereafter, WD) must be high to justify the short recurrence time. In fact, a certain pressure must be built on the envelope in order to trigger a thermonuclear runaway due to $\mathrm{CNO}$ burning, the commonly accepted mechanism causing nova outbursts. The outburst pressure is reached sooner when $\dot{m}$ is higher.

Generally, the different authors agree that several outburst and evolutionary properties of RN are explained by the characteristics of the secondary star. In Table 1, T Pyx, IM Nor and CI Aql are short period systems, with a main sequence or near-main-sequence secondary. U Sco, V394 CrA, and most likely V2487 Oph host an evolved secondary, with periods of the order of a day. Finally, T CrB, RS Oph, V745 Sco and V3890 Sgr are wide binaries with a red giant in an orbit with a period of the order of few years. It is clear that RN with evolved secondaries have very fast outbursts, and generally there is evidence of very massive WDs. The outbursts of RN with few hours periods, can be slow, and probably $\mathrm{m}(\mathrm{WD})$ can span a wider range.

A proposal by Schaefer (2010) to divide RN into binaries with outburst triggered by irradiation 
induced mass transfer and "other systems" probably does not well fit the RN observations. As I discuss in Section 4, the supersoft X-ray phase of the RN candidates for irradiation induced mass transfer is in fact very short, and it is hard to conceive that it would be responsible for driving renewed mass transfer quickly after the eruption. Calculations for irradiation induced mass transfer triggering novae were done by Kovetz et al. (1988).

A serious problem in studying the Galactic RN is that of the poorly known distances. The fact that the MMRD does not hold with short recurrence time objects is a problem. We are dealing with a large range of distances in the sample of Table 1 . While T CrB may be at only $900 \mathrm{pc}$, based on the magnitude and spectral type of the giant companion, we know that U Sco is likely to be at $12 \mathrm{Kpc}$; this RN, V394 CrA and V2487 Oph are all least $10 \mathrm{kpc}$ away from us. Clearly, all the distances to these objects have been estimated with a large uncertainty. I note that Sokoloski et al. (2013) proposed to revise the commonly accepted distance of RS Oph of $1.6 \mathrm{kpc}$ to $4.8 \pm 0.5 \mathrm{kpc}$. However, this would make the supersoft X-ray source largely super-Eddington for an extended period of time, which seems very unlikely. At $4.8 \mathrm{kpc}$ the companion does not fill its Roche Lobe, and RS Oph is thought to have an accretion disk. As Joanna Mikolajewska discussed at this conference, this may be explained by a more complicated mechanism of disk formation in symbiotics than "simple" Roche Lobe overflow. In any case, we have to wait for the second GAIA release to obtain well constrained distance measurements to these novae. Accurate distances will imply significant improvement in modeling RN.

\section{Recurrent Novae in other Local Group galaxies}

More than 40 novae or nova candidates have been observed in the Magellanic Clouds, and among them 3 RN are known: YY Dor or N1937/2004, N LMC 1968/1991, N LMC 1971b/2009. In addition, N LMC 2012 is also a candidate RN.

In M31, 964 assessed or suspected nova eruptions have been recorded before the date of publication of Shafter et al.'s 2015 paper. This number of course may include up to 10\% spurious objects, mainly M31 black hole systems, and rare foreground dwarf novae. We know $12 \mathrm{RN}$ in this galaxy (with an additional 4 candidates), but the estimated discovery efficiency is only $19 \%$ (Shafter et al. 2015). Many RN (at least the ones without red giants companions) are not intrinsically very luminous at maximum, and can easily be missed. In Shafter et al. (2015) we find that the estimated RN recurrent nova rate is such that it would contribute by only $2 \%$ to the SNe Ia rate observed in all galaxies. However, this is true only in the assumption that the sub-Chandrasekhar explosions are not frequent, and in another presentation at this conference I show evidence in favor of the opposite.

One of the most interesting findings of extragalactic RN searches has been the discovery of Nova M31 2008 12a, which has a very short recurrence time, of the order of half a year (or less; Henze et al. 2015). Only by looking in a wider volume were we able to find such an extreme object, proving how short the recurrence time of a thermonuclear runaway can be, still with mass ejection. The theory predicts that at some very short recurrence time the layer in which the burning occurs does not have sufficient pressure to cause the conditions of mass ejections, and degeneracy is lifted before a wind from the WDs is produced. When this occur, there is a possibility of avoiding mass loss, reaching the Chandrasekhar mass. 

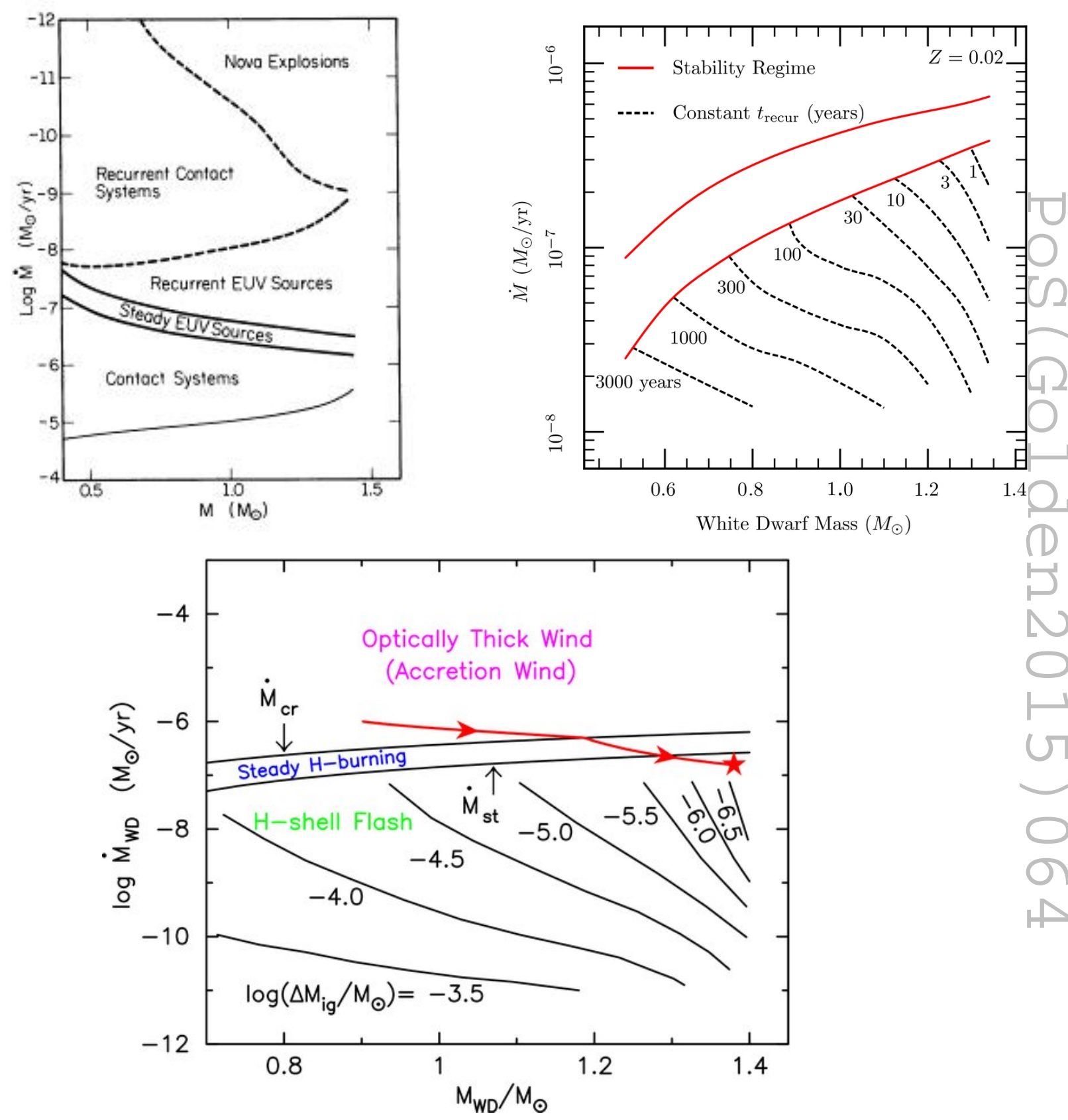

Figure 1: On top left panel, a figure from Fujimoto (1982) is reproduced, on the right top panel, Fig. 1 of Wolf et al. (2013) is shown, and finally on the bottom panel a figure by Hachisu et al. (2010). These figures illustrated the similar results of the model calculations regarding the stability regime in the m(WD)- $\dot{m}$ plane. 


\section{Correlations between observed parameters}

In its Comprehensive Photometric Histories of all Known RN, Schaefer (2010) finds no obvious correlation between the speed class of RN and their orbital period. Because the speed class according to the models is correlated with $\dot{m}$, this implies that $\dot{m}$ isn't probably correlated along with the orbital period of a RN.

Another interesting theoretical correlation is the one between $\dot{m}$ and $\mathrm{m}(\mathrm{WD})$. Several authors predict the loci of constant recurrence time in the m(WD) vs. $\dot{m}$ plane. In Fig. 1 I show results obtained over a many-years range by Fujimoto (1982), Hachisu et al. (2010) and Wolf et al. (2013). there are observational diagnostics, like the time for a decay by 2 magnitudes in optical and the ejecta velocity, tha allow to roughly estimate $\mathrm{m}(\mathrm{WD})$ in several RN. U Sco, RS Oph, T CrB, V745 Sco are all thought to host WDs close to the Chandrasekhar limit.

\section{From short recurrence time to steady burning}

In Fig. 1 the three plots taken from the literature illustrate the m(WD) vs. $\dot{m}$ parameter space in different ways. Regardless of the details of the calculations, Fujimoto was the first author who explored the parameters' space. Several quantities, like the opacities, have been updated in the calculations, but I show all three figures because they are useful to understand the different possible outcomes. Fig. 1 from Wolf et al. (2013) and Fig. 2 from Fujimoto et al. (1982) show that there is a continuum in the combination of parameters $\dot{m}$ and $\mathrm{m}(\mathrm{WD})$, from shorter and shorter recurrence times to steady burners without mass ejection. In the parameters' space shown in Fig. 1 of Wolf et al. the steady burners occur between the red lines, and if no helium flash occurs before m(WD) increases to a value that leads to an explosion, there is a type Ia SN event. Steady burning may also occur above the upper red line, if it is regulated by a wind like suggested by Kato et al. (2010, see the red line of Fig.3). In Fig.2, the "recurrent and steady EUV sources" are what we rather found to be "supersoft X-ray sources", either with a thermonuclear flash without mass loss, or with quite burning without thermonuclear runaways.

In "real life" we do not expect constant $\dot{m}$ and we do not expect a fixed time between thermonuclear runaways. Shaviv et al. (2014) have done new research by evolving the WD together with its companion and checking how irradiation causes feedback on the secondary and changes mass transfer. It turns out that there may be a short RN phase separated then by thousands of years from the next outburst, and the next occurrence of RN behaviour. This may imply that only steady burners can reach the Chandrasekhar mass.

\section{X-ray observations of RN}

X-ray observations are a powerful mean of discovering the physics of nova systems. Novae in outburst, and RNe in particular, are among the most luminous objects in the X-ray sky. Not only the central source, but also the ejecta are very X-ray luminous. This is especially true for RN with a red giant companion, in which the previous red giant wind must be strongly shocked by the impact of the ejecta, as in the typical case of the RS Oph 2006 outburst (Nelson et al. 2008). However, shocked ejecta must also exist in all novae and RN, even in those without a red giant wind or planetary nebula material. Probably there are colliding winds. 
Only through the X-ray window, can we obtain a glimpse of the H-burning nova WD after the star contracts to almost pre-outburst dimension, while hydrogen is still burning at the bottom of the accreted envelope, with only a thin atmosphere on top. The more massive the WD, the more compact it is, and the hotter is the atmosphere. Two problems have arisen:

1. Because the atmospheric effective temperature $T_{\text {eff }}$ is not high to produce absorption lines of $\mathrm{Ne}$ and $\mathrm{Mg}$, it is more difficult than expected to separate $\mathrm{CO}$ WDs from $\mathrm{NeO}$ WDs. A clue from the atmosphere may be the Ne absorption edge at $\simeq 62 \AA$, cutting off the flux on the low side (this is only measurable with the Chandra Low Energy Transmission Grating). Some of the absorption lines that require the high abundances of certain intermediate atomic number elements produced in the $\mathrm{CNO}$ cycles branches on $\mathrm{NeO}$ WDs are more difficult to measure. We actually learn more about the WD composition from the ejecta, even in the X-ray spectrum of the ejecta.

2. The absorption lines are not at rest, the are blue-shifted with typical velocities exceeding a thousand $\mathrm{km} \mathrm{s}^{-1}$. This means that mass loss continues even during photospheric contraction, most likely in a high velocity, low $\dot{m}$ wind, even after the "nominal" end of mass loss at higher mass loss rate. Most probably, what happens is a receding photosphere with an expanding atmosphere, therefore we cannot determine the effective gravity, and until the residual wind ceases we only have a lower limit on the mass from $\mathrm{T}_{\text {eff }}$.

A very important result the determination of $\mathrm{T}_{\text {eff }}$ by fitting static atmospheric models with "artificially" blue shifted features (Rauch et al. 2010); this is because we do not have a suitable grid of expanding atmosphere models with ad-hoc abundances for novae. Such models are more difficult to calculate (see van Rossum 2012). As Fig. 2 shows, the derived $T_{\text {eff }}$ correlates well with $t_{2}$, which is almost linearly dependent on $\mathrm{m}$ (WD) according to the theory (see figures in Wolf et al. 2013). Only one $\mathrm{RN}$ does not seem to fit this relationship: the outlier, not shown in the figure, is $\mathrm{T}$ Pyx. For this nova we only have an upper limit of $420,000 \mathrm{~K}$ for $\mathrm{T}_{\text {eff }}$, because the $\mathrm{X}$-ray spectrum of the ejecta was very luminous compared with that of the WD, but probably the real value was quite close to this limit - $\mathrm{t}_{2}$ was long, 32 days.

In two RN, T Pyx and U Sco, the measured X-ray luminosity was too low to be due to the whole WD atmosphere, which should reach near-Eddington luminosity. Such a puzzle has not been found in any classical novae, where the X-ray luminosity so far is consistent with the models. Moreover, in both T Pyx and U Sco there was an orbital modulation of the supersoft X-ray flux, which was difficult to understand given the central source (WD) size at the observed stage (it had almost shrunk to re-outburst dimensions). The root cause of the low luminosity seems to be different in the two cases. For T Pyx the unabsorbed X-ray luminosity of about $1.5 \times 10^{35} \mathrm{erg} \mathrm{s}^{-1}$, corresponding to a bolometric luminosity of $10^{36} \mathrm{erg} \mathrm{s}^{-1}$ was a factor of $\simeq 30$ lower than expected (Tofflemire et al. 2013). Optical spectra, shown in Fig. 3, indicate that the reason is likely to have been a partial obscuration by mass ejection without spherical symmetry. The nebular [O III] lines have a complex structure that can be attributed to bipolar outflow. Shore et al. (2013) also inferred mass ejection in bipolar flows from their optical spectra. Two more things are peculiar in T Pyx, namely the short duration of the supersoft X-ray phase, which is not consistent with the measured $\mathrm{T}_{\text {eff }}$ (in other novae it is inversely dependent on $\mathrm{T}_{\text {eff }}$, as predicted by the models) and the long nebular phase. Both phenomena seem to imply that this RN ejects little mass, and in Tofflemire et al. (2013) we have suggested that the nova retains a large part of the accreted material. The cause of the low X-ray (and bolometric) luminosity in U Sco, which is a high inclination system, seems 
to be different. It is very likely that we were not observing the WD at all, but a more extended, lower luminosity emission due to Thomson scattering (Ness et al. 2012, Orio et al. 2013). In this case, the orbital variability is also explained since the accretion disk must have been present in the outburst or quickly reconstructed.

U Sco was observed once with Chandra and twice with XMM-Newton. While initially it showed emission and absorption, eventually it displayed only emission lines, whose prominence indicates that they cannot have originated "near the WD", so that an apparent P-Cyg profile in the early spectrum is due to the superimposition of an atmospheric absorption feature and an emission feature in the ejecta. As the ejecta expanded the emission lines became broader and the absorption lines were engulfed in the overlapping broad emission lines due to expanding material.

A very interesting set of $4 \mathrm{X}$-ray grating spectra at different epochs were obtained for the RN LMC 2009 with the XMM-Newton RGS. The spectrum initially resembled that of U Sco even if the nova became X-ray luminous only after 3 months, not after a week like U Sco. The emission spectrum gradually decreased in importance and the luminous atmospheric emission emerged. This example shows that, even if the X-ray spectrum and the relative prominence of emission lines spectra and atmospheric spectra are often determined by the nova inclination, as suggested by Ness et al. (2013), the inclination cannot be the only cause of one type of spectrum. Novae evolve during the outburst and the relative importance of the two type of spectra change during the post-outburst evolution, depending on the quantity of ejected mass and $\mathrm{m}(\mathrm{WD})$.

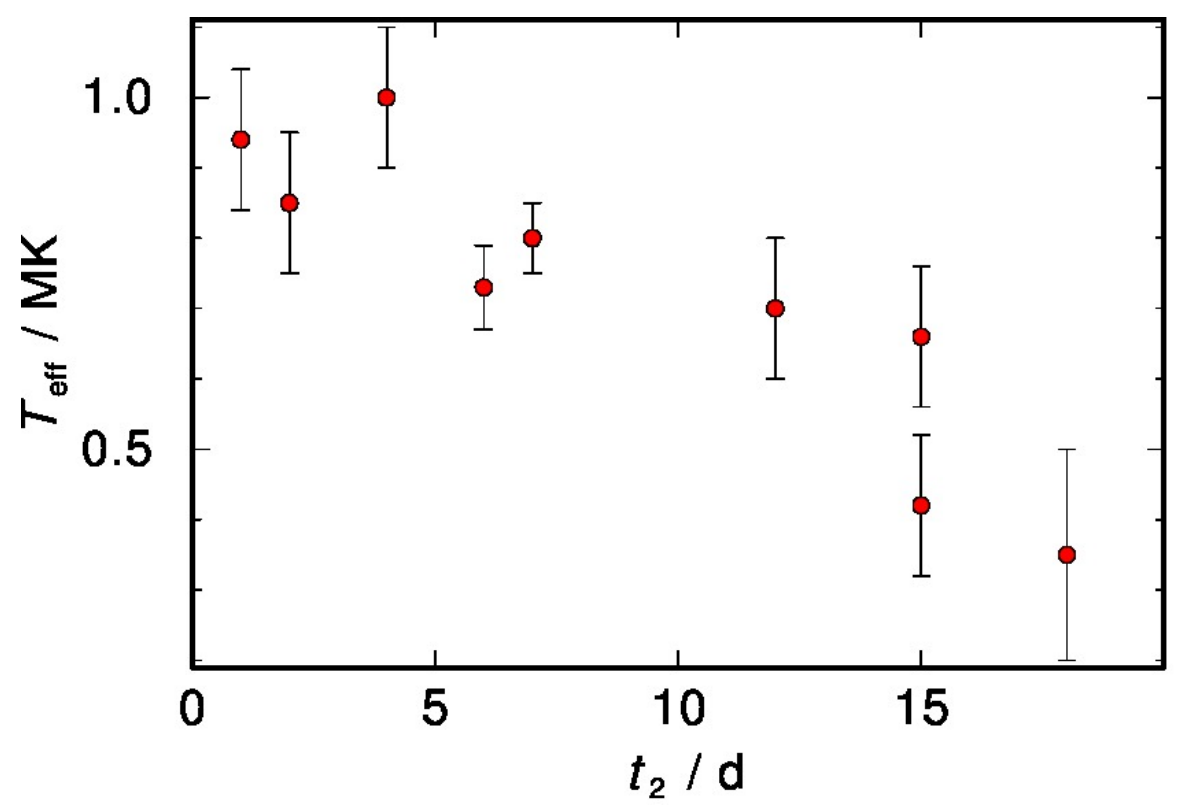

Figure 2: $\mathrm{T}_{\text {eff }}$ in $10^{6} \mathrm{~K}$ vs. $\mathrm{t}_{2}$ (in days) derived with atmospheric models (Rauch et al. 2010) for, from left to right, U Sco, RS Oph, V2491 Cyg, V4743 Sgr, HV Cet, V5116 Sgr, KT Eri, N LMC 1995, GQ Mus. We used grating spectra for all except N LMC 1995 and GQ Mus. Details and references can be found in Orio, 2012. 


\section{X-ray flux modulation as a new type of diagnostic}

The supersoft X-ray flux of several WDs in novae is modulated with short periods or semiperiods. Two RN have shown very short periods, $33.2 \mathrm{~s}$ in N LMC 2009a, and $34.9 \mathrm{~s}$ in RS Oph. A period of $35 \mathrm{~s}$ in a Galactic RNe candidate, KT Eri. Details and full references can be found in Ness et al. (2015). There is another range of periods observed in classical novae, of the order of tens of minutes. The periods of the order of a minute are shared by the classical nova V339 Del (54.1 s) and the steady burner CAL 83 (66.8 s). Possible explanations proposed for these short periods are the WD rotation period, which can be very short in massive, accreting WDs, spun-up by accretion, but this requires a non-homogeneous atmosphere. It is more likely that the modulations are due to non-radial g-mode oscillations caused by the $\varepsilon$ mechanism like in hot PG 1059 stars (Corsico et al. 2009).

\section{X-ray observations at quiescence}

If the X-ray flux at quiescence originates in the boundary layer of a disk, or from accretion onto polar caps of a magnetized WD, it should increase with $\dot{m}$. However, RNe are not particularly $\mathrm{X}$-ray luminous compared with other WDs. This is in contrast with other cataclysmic variables that are thought to be accreting at high $\dot{m}$, the nova-like, demonstrating that there are indeed systems with a correlation between $\dot{m}$ inferred from other evidence than X-rays, and that inferred from the X-ray flux (Zemko et al. 2014 and references therein).

For RS Oph, from X-ray observations $\dot{m}<2 \times 10^{-8} \mathrm{~m}_{\odot}$ year $^{-1}$ has been derived, which is inconsistent with the models, that require higher $\dot{m}$ (Nelson et al. 2011). This a problem that should still be solved.

Another rather mysterious phenomenon is the flux decrease at late phases or at initial stages of quiescence, at constant $\mathrm{T}_{\mathrm{eff}}$ in the soft component. This has been inferred for $\mathrm{T}$ Pyx (Tofflemire et al. 2013), V2491 Cyg (Page et al. 2010, Zemko et al. 2015), V4743 Sgr (Zemko et al. 2016). Is there a shrinking of the area of supersoft X-ray emission before full cooling takes place?

\section{Conclusions}

$\mathrm{RNe}$ are a very diverse group of objects with different characteristics and the only common features are the small amplitude and short recurrence time (prove that the TNR theory has a strong basis). However, there still isn't sufficient observational proof that they are all accreting at high rate.

The RN phase may well not be a final phase in the evolution of a RN binary, it may be followed by another classical nova phase. For future discoveries rely on new instruments and multiwavelength observations. The X-ray range has yielded several observational surprises, including the shocked ejecta, the short period oscillations, and it allows probing the WD atmospheric temperature, which in turn depends on $\mathrm{m}(\mathrm{WD})$.

We need to look at least in the whole Local Group to have sufficient statistics to better understand RN. These intriguing binaries are important and interesting even if they are not going to end as SNe Ia progenitors. I am accepting bets about which known RN will explode next in the Galaxy... does anyone want to take thiis challenge? 


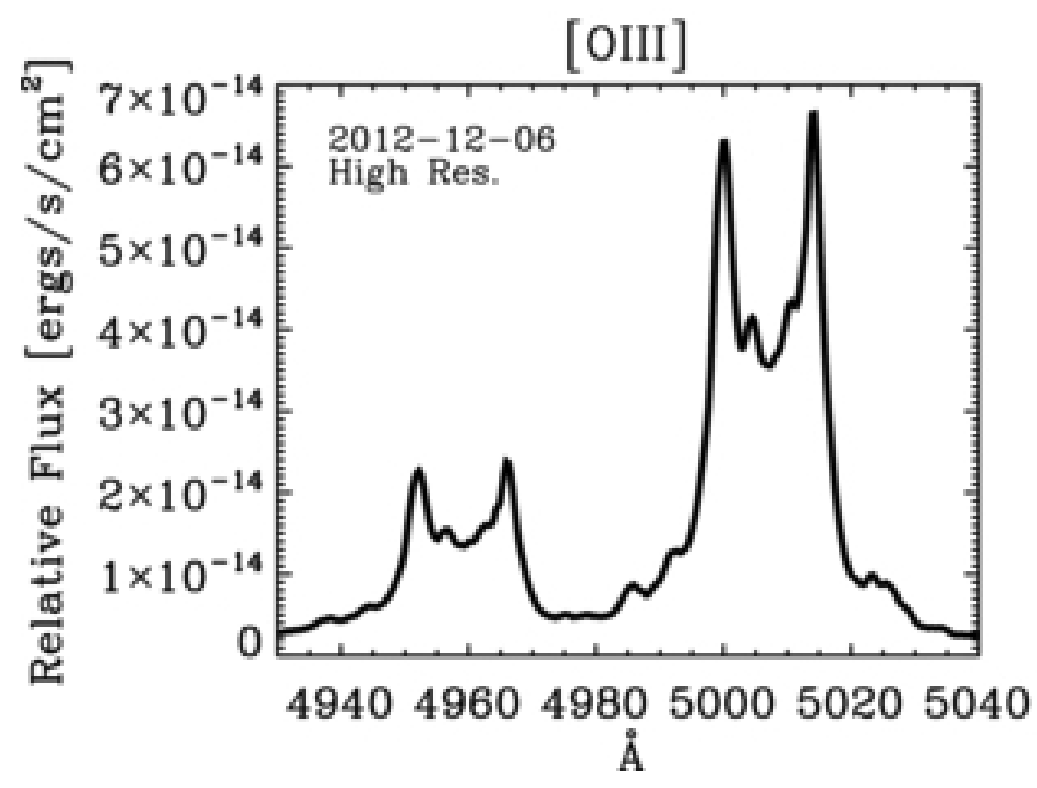

Figure 3: SALT optical spectrum of T Pyx in the nebular phase. The [O III] lines at 4960 and $5007 \AA$ show the bipolar morphology discussed in the text (from Tofflemire et al. 2013).

\section{References}

[1] Corsico, A.H., et al. 2009, ApJ, 701, 1008

[2] Fujimoto, M.Y. 1982, ApJ, 257, 767

[3] Hachisu. I., Kato, M. \& Nomoto, K. 2010, ApJ, 724, L212

[4] Henze, M., Darnley, M.J., et al. 2015, A\&A, 582, L8

[5] Nelson, T., Orio, M., et al. 2008, ApJ, 673, 1067

[6] Nelson, T., Mukai, K., Orio, M., Luna, G.J.M., \& Sokoloski, J.L. 2011, ApJ, 773, 7

[7] Ness, J.-U. et al. 2012, ApJ, 745, 43

[8] Ness, J.-U., et al. 2015, A\&A, 578, 39

[9] Orio M. 2012, BASI, 40, 333

[10] Page, K. 2010, MNRAS, 416, 121

[11] Rauch, T., Orio, M. et al., 2010, ApJ, 717, 363

[12] Shafter, et al. 2015, ApJS, 216, 34

[13] Schaefer, N. 2010, ApJS, 187, 275

[14] Shaviv, G., Idan, U., \& Shaviv, N.J. 2014, arXiv 1411.5300

[15] Sokoloski, J., Crotts, A.P.S., Stephen, L., \& Uthas, H. 2013, ApJ, 770, L33

[16] Tofflemire, B.i, Orio, M., et al. 2013, ApJ, 779, 22

[17] van Rossum, D. 2012, ApJ, 756, 31 
[18] Williams, R. 2012, 144, 98

[19] Wolf, W., Bildsten, L., Borrks, J., \& Paxton, B. 2013, ApJ, 777, 136, 2013

[20] Zemko, P., Orio, M., Mukai, K., \& Shugarov, S. 2014, MNRAS, 445, 869

[21] Zemko, P., Mukai, K., \& Orio, M. 2015, ApJ, 807, 61 S. 201

[22] Zemko, P., Orio, M., Mukai, K., Bianchini, A., Ciroi, S., \& Cracco, V. 2016, MNRAS, 460, 2744 